\title{
Fuzzy Logic based Square Root Information Filter for Image Centroid Tracking
}

\author{
Parimala ${ }^{1}$, P., Raol ${ }^{2}$, J. R. \\ ${ }^{I}$ Asst. Prof., Dept. of Telecommunications Engg, MSRIT, and Research Scholar, Jain University, Bangalore. \\ ${ }^{2}$ Prof. Emeritus, MSRIT, Bangalore.
}

\begin{abstract}
In this paper fuzzy logic based square root information filtering (SRIF) algorithm for target image centroid tracking is presented proposed. It combines certain merits of the fuzzy logic (FL) and (SRIF) algorithm for target tracking. A data fusion study in the context of image-centroid fusion is also presented with certain parametric studies. The performance metrics are evaluated by utilizing sequence of synthetic images. Implementation is carried out in MTALAB.
\end{abstract}

Keywords: Centroid tracking algorithms (CTA), square root information filter, fuzzy logic, performance metrics

\section{INTRODUCTION}

An object should be assigned with correct coordinates/position of the considered image. In order to determine the coordinate of the object-image, the centre of area of the target is chosen as the representative position. This is estimated by a parameter called centroid of the target[1].

Information filtering (IF) is a more direct way of dealing with target tracking and multi-sensor data fusion problems than the conventional covariance based KF. However, the IF could be sensitive to computer round-off/quantization errors. This would degrade the tracking performance of the filter. The square root information filter (SRIF) offers a solution to this problem of numerical accuracy and stability of the filtering algorithm [2]. Fuzzy logic uses an interval of real numbers between zero and one to develop logic as a basis for rules of inference. Particularly the fuzzified version enables computers to make decisions using fuzzy reasoning rather than exact. The factors like low signal-to-noise (SNR) ratio in the acquired image/s, low contrast, presence of background clutter and/or false alarms and partial occlusion of the target image necessitate the use of efficient, accurate, and numerically stable filtering algorithms for image-centroid tracking and image fusion [1]. Certain studies on square root type factorization filtering algorithms for state estimation and target tracking have been carried out [6],[7] [11], but in a limited way. In this paper fuzzy logic augmented SRIF is presented for image-centroid tracking and fusion.

\section{FUZZY LOGIC BASED IMAGE TRACKING}

Fuzzy logic is a form of many-valued logic. Fuzzy logic has been applied to many fields, from control theory to robotics. A fuzzy set A in the universe of discourse $\mathrm{U}$ can be defined as a set of ordered pairs,

$$
A=\left\{\left(x, \mu_{A}(x)\right) \mid x \in U\right\}
$$

Where $\mu_{A}$ is called the membership function of $A$ and $\mu_{A}$ is the grade (or degree) of membership of $x$ in $A$, which indicates the degree that $x$ belongs to $A$. The membership functions used for the parametric study of the tracking algorithms could be: i) triangular membership function, or ii) ttrapezoidal membership function, or any other suitable function. Fuzzy if-then rules are expressed in the form, IF A THEN B, where A, and B are labels characterized by appropriate membership function [1][3]. The fuzzy inference system (FIS) uses fuzzy reasoning to map an input to the output.

Basically a FIS consists of:

- Fuzzification interface which transforms the crisp inputs into degrees of match with linguistic values

- Rule base containing a number of fuzzy if-then rules

- Database which defines the membership functions of the fuzzy sets used in the fuzzy rules

- Decision-making unit which performs the inference operations on the rules

- Defuzzification interface which transforms the fuzzy results of the inference into a crisp output.

The object of interest from the background is identified using particle segmentation. Target is detected in the received image sequences in two steps 
[1]: i) binary conversion of grey level using lower and upper threshold limits of the target, thresholds are identified using histogram of the images acquired by the sensors; and ii) nearest neighbour technique is used to detect pixels of the target which are grouped into clusters.

The centroid of clustered pixels of object is determined using the following formula[3]:

$\left(\mathrm{x}_{\mathrm{c}}, \mathrm{y}_{\mathrm{c}}\right)=$

$\frac{1}{\sum_{\mathrm{i}=1}^{\mathrm{n}} \sum_{\mathrm{j}=1}^{\mathrm{m}} \text { Iij }}\left[\sum_{\mathrm{i}=1}^{\mathrm{n}} \sum_{\mathrm{j}=1}^{\mathrm{m}} \mathrm{iI}(\mathrm{i}, \mathrm{j}), \sum_{\mathrm{i}=1}^{\mathrm{n}} \sum_{\mathrm{j}=1}^{\mathrm{m}} \mathrm{jI}(\mathrm{i}, \mathrm{j})\right]$

In (2), Iij or I(i,j) is the intensity of the pixel with co-ordinates $(i, j)$ and $n, m$ are the dimensions of the cluster. The 'regionprops' function in MATLAB is used to find this centroid of the target image. Image tracking system needs lower computational cost in filtering, efficient data association scheme, and numerically accurate and stable algorithm. Squareroot type algorithm is more suitable for such requirements.

In a centroid tracking algorithm (CTA), the determination of a moving object's position and velocity from a noisy time series of images, is an estimation problem. A suitable state space model for centroid representation is given by [1]

$$
\begin{aligned}
& x(k+1)=\phi x(k)+G w(k) \\
& z(k+1)=H x(k)+\mathrm{v}(\mathrm{k})
\end{aligned}
$$

In (3), and (4), $x$ is a state vector that contains the image-centroid coordinates of a target, $\mathrm{z}$ is the vector of observables (image-centroid measurements), and $\mathrm{w}($.$) , and \mathrm{v}($.$) are process and$ measurement noises with zero means and covariance matrices Q, and $\mathrm{Rm}$ respectively. These noise processes are assumed to be white and Gaussian.

\section{CTFSRIF ALGORITHM FOR IMAGE TRACKING}

A widely used form of the algorithm is square root information filter (SRIF). If $\tilde{\mathrm{x}}$ is an a priori estimate of $\mathrm{x}$, having a priori information matrix $\mathrm{P}^{-1}$, then a priori state information pair is given by $\left(\tilde{\mathrm{x}}, \mathrm{P}^{-1}\right)$. The information matrix $\mathrm{P}^{-1}$ can be factored as $\left(\mathrm{R}^{\mathrm{T}}, \mathrm{R}\right)$. After simplifying the cost function, the combined system can be represented by [2][4]

$$
\left[\begin{array}{l}
\tilde{y} \\
Z
\end{array}\right]=\left[\begin{array}{l}
\tilde{R} \\
H
\end{array}\right] x+\left[\begin{array}{l}
\tilde{V} \\
V
\end{array}\right]
$$

Thus, the a priori information forms a data equation similar to the measurement equation, and it is considered as an additional measurement. The square root information pair and the existing measurements are put in the following form and orthogonal transformation is applied to obtain the Least-square (LS) solution

$T\left[\begin{array}{cc}\tilde{R}(k-1) & \tilde{y}(k-1) \\ H & z(k)\end{array}\right]=\left[\begin{array}{cc}\hat{R}(k) & \hat{y}(k) \\ 0 & e(k)\end{array}\right](6)$ where $\mathrm{T}$ is the Householder transformation matrix, $\mathrm{e}(\mathrm{k})$ is a sequence of residuals. Since, $\mathrm{e}(\mathrm{k})$, the residuals are available from the previous cycle (or the initial conditions); separate the $\mathrm{e}(\mathrm{k})$ into $\mathrm{e}_{\mathrm{x}}(\mathrm{k})$, and $\mathrm{e}_{\mathrm{y}}(\mathrm{k})$ and obtain the following derivatives:

$$
\dot{e}_{x}=\frac{\left\{e_{x}(k+1)-e_{x}(k)\right\}}{T} \dot{e}_{y}=\frac{\left\{e_{y}(k+1)-e_{y}(k)\right\}}{T}
$$

Then, form the following FL correlation function:

$$
r_{f l c}=f_{m}(k+1)=\left[f_{m x}(k+1) \quad f_{m y}(k+1)\right]
$$

The function $\mathrm{f}($.$) is the FMF, which is the$ fuzzy logic correlator (FLC). Inputs to the FLC are the residuals and their derivatives, and the output is $\mathrm{r}_{\mathrm{flc}}$. The updated information pair $(\hat{y}(k), \hat{R}(k))$ is generated. The process of estimation can be continued by including next measurement.

$$
\hat{x}=\hat{R}^{-1} \hat{y}
$$

Where $\hat{x}$ is filtered state in covariance domain; $\hat{y}(k)$ serves as priori estimate $\tilde{y}(k-1)$ for the next measurement update. $\hat{R}(k)$ serves as priori estimate $\tilde{R}(k-1)$ for the next measurement update, $\mathrm{z}(\mathrm{k})$ is the current measurement. In the measurement/data update part, it does not need the computation of the Kalman gain, since, now it uses the measurement/data update part of the SRIF, and hence, it is the gain free filter; the main reason is that the information state yf is now directly available from the orthogonal transformation. It retains the merits of the CTSRIF [10].

\section{IMAGE FUSION USING CTFSRIF}

A system with two sensors models H1 and $\mathrm{H} 2$ is used and their measurements can be fused at data level [5][6]. The process results in the state estimate due to the two sensor data fused.

$T(k)\left[\begin{array}{cc}\tilde{R}_{f}(k-1) & \tilde{y}_{f}(k-1) \\ H_{1}(k) & z_{1}(k) \\ H_{2}(k) & z_{2}(k)\end{array}\right]=\left[\begin{array}{cc}\hat{R}_{f}(k) & \hat{y}_{f}(k) \\ 0 & e(k)\end{array}\right] ; k=1, \ldots$,

Here, $\widehat{y} f$ is the fused information state. Since, now yf is available, this Rf and yf are used in equation (10) for CTFSRIF fusion. Measurement updating is carried out and updated $\mathrm{xf}$ (for each time step) is calculated using

$$
x f=\operatorname{inv}(R f) y f
$$

These values are used in the time propagation part of CTFSRIF to obtain optimum tracking results.

\section{COMPARISON OF CTSRIF AND CTFSRIF ALGORITHM}


A set of 50 synthetic images that represents target environment is considered for the evaluation of these algorithms. For centroid computation formula (2) is used in MATLAB. Each image frame is of the dimension $64 \times 64$ with the target size fixed with a dimension of $9 \times 9$. The image constitutes an object and its surrounding along with noise that is uniformly distributed. The measurement noise is assumed to be Gaussian with zero mean and covariance $\sigma 2$. Intensity of the grey level image varies in the range 0 to 255 . Target image intensity has a mean and std $(100,10)$; while target background intensity has a mean and STD $(50,50)$; $\{$ these can be varied based on the study, TGBSTD . The measured centroid of the given synthetic image is determined. The other input parameters for the tracking algorithm are:

Measurement model/matrix: $H=\left[\begin{array}{llllllll}1 & 0 & 0 & 0 ; & 0 & 0 & 1 & 0\end{array}\right]$;

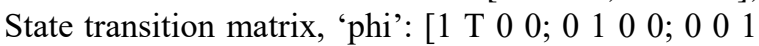
T; $\left.\begin{array}{llllll}0 & 0 & 0 & 1\end{array}\right]$;

Measurement noise variance: $\mathrm{Rm}=0.5$ (is varied based on the study); Process noise coefficient matrix: $\mathrm{G}=\left[\mathrm{T}^{2} / 2 \quad 0 ; \mathrm{T} 0 ; 0 \mathrm{~T}^{2} / 2 ; 0 \mathrm{~T}\right]$; Process noise covariance: $\mathrm{Q}=0.00001$ (is varied if needed); Track scan (sampling interval/period,T): 1 sec.; The initial states $\{\mathrm{x}(0), \mathrm{y}(0)\}=(10,10)$, with constant initial velocity of $1 \mathrm{~m} / \mathrm{s}$ in both the coordinates. The performance metrics \% fit error (PFE) and root mean square error for position (RMSPE) and velocity (RMSVE) are evaluated as follows:

$\mathrm{PFE}=\%$ fit error

$=\mid$ state or measurement error $\mid * 100$

$$
\operatorname{RMSPE}=\sqrt{(\text { mean(xperr2 }+ \text { yperr2 }))}
$$

\subsection{IMAGE CentroId Tracking SRIF ALgORITHMS}

Table $1 \& 2$ give the performance metrics of CTSRIF and CTFSRIF filter for different background target image noise standard deviation (TGNSBSTD) and different target image noise standard deviation ( TGNSTD).

Table 1: Metrics using CTSRIF/CTFSRIF for

\begin{tabular}{|l|l|l|}
\multicolumn{4}{c}{ TGNSTD $=5$} \\
\cline { 2 - 3 } $\begin{array}{l}\text { Parameter } \\
(*) \text { Metrics } \\
\text { (\%fit errors) }\end{array}$ & CTSRIF & CTFSRIF \\
\hline PFEx & 0.6130 & 0.5918 \\
\hline PFEy & 0.7113 & 0.6851 \\
\hline RMSPE & 0.3512 & 0.3469 \\
\hline RMSVE & 0.0315 & 0.0315 \\
\hline
\end{tabular}

Table2: Metrics using CTFSRIF/CTFSRIF for TGNSTD $=5$

\begin{tabular}{|l|l|}
\hline Parameter & TGNSBSTD $=\mathbf{1 0 0}$ \\
\hline
\end{tabular}

\begin{tabular}{|l|l|l|}
\hline $\begin{array}{l}(*) \text { Metrics } \\
(\% \text { fit } \\
\text { errors })\end{array}$ & CTSRIF & CTFSRIF \\
\hline PFEx & 0.4145 & 0.3822 \\
\hline PFEy & 0.4957 & 0.4833 \\
\hline RMSPE & 0.2417 & 0.2361 \\
\hline RMSVE & 0.0277 & 0.0277 \\
\hline
\end{tabular}

It is seen that there is not much of trend of the performance metrics with respect to the STDS. However, it is established that CTFSRIF performs somewhat better than CTSRIF in a similar centroid tracking task.

\subsection{Image Centroid Fusion Using SRIF Algorithms}

Two sensor images with different target noise standard deviations 1 and 5 (and with the target background noise standard deviation as 100) are considered. Using the measurement level fusion (MLF) both the centroids of images are fused using CTSRIF as in equation (9) filter and CTFSRIF filter logic as in equations (7), (8) and (9). Table $3 \& 4$ show the performance metrics of image-centroid fusion CTSRIF and CTFSRIF with TGNSBSTD $=100$. Figure 1 shows measurement level fusion performance with CTSRIF and CTFSRIF for target noise std as $1 \& 5$ and background noise std of 100. The MLF is found to be quite satisfactory, and CTFSRIF is slightly better than CTSRIF.

Table 3: Metrics of image-centroid fusion using CTSRIF for TGNSTD $=1 \& 5$

\begin{tabular}{|l|l|l|l|}
\hline $\begin{array}{l}\text { Parameter } \\
(*) ; \text { Metric }\end{array}$ & Filter & \multicolumn{3}{|l|}{} \\
\cline { 2 - 4 } $\begin{array}{l}\text { s } \\
(\% \text { FE })\end{array}$ & $\begin{array}{l}\text { CTSRIF1 } \\
\text { TGNSTD }=\end{array}$ & $\begin{array}{l}\text { CTSRIF2 } \\
\text { TGNSTD } \\
=5\end{array}$ & $\begin{array}{l}\text { CTSRIF } \\
\text { MLF }\end{array}$ \\
\hline PFEx & 0.3778 & 0.4145 & 0.3895 \\
\hline PFEy & 0.6652 & 0.4957 & 0.5738 \\
\hline RMSPE & 0.2879 & 0.2417 & 0.2604 \\
\hline RMSVE & 0.0261 & 0.0277 & 0.0277 \\
\hline
\end{tabular}

Table 4: Metrics of image-centroid fusion using

\begin{tabular}{|c|c|c|c|}
\hline \multirow{3}{*}{$\begin{array}{l}\text { Parameter } \\
\left({ }^{*}\right) ; \text { Metrics } \\
(\% \mathrm{FE})\end{array}$} & \multicolumn{3}{|l|}{ Filter } \\
\hline & CTFSRIF1 & CTFSRIF2 & CTFSRIF \\
\hline & TGNSTD $=1$ & TGNSTD $=5$ & MLF \\
\hline PFEx & 0.3658 & 0.3822 & 0.3775 \\
\hline PFEy & 0.6552 & 0.4833 & 0.5638 \\
\hline RMSPE & 0.2876 & 0.2361 & 0.2600 \\
\hline RMSVE & 0.0261 & 0.0277 & 0.0277 \\
\hline
\end{tabular}
CTFSRIF for TGNSTD $=1 \& 5$ 


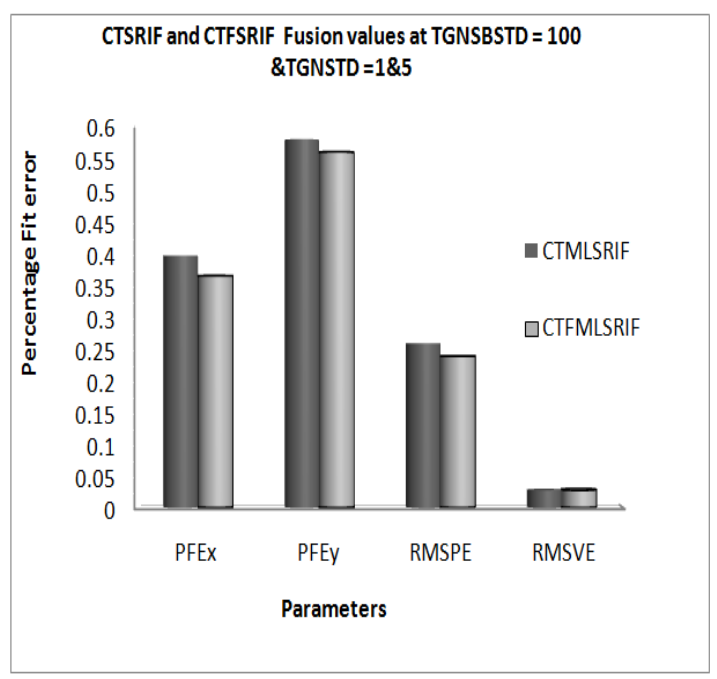

Figure 1 MLF for CTSRIF and CTFSRIF

\section{CONCLUSIONS}

The image-centroid tracking and fusion using fuzzified square root type filtering algorithm CTFSRIF is evaluated with synthetic images generated. The performance metrics and plots show that the CTFSRIF gives slightly better performance than CTSRIF in tracking and fusion of images. Also, the performance of these algorithms is nearly robust.

\section{REFERENCES}

[1] Parimala, P., and Raol, J. R. Fuzzy Logic and ANFIS based Image Centroid Tracking and Filtering Schemes International Journal of Enhanced Research in Science, Technology \& Engineering ISSN: 23197463, Vol. 5 Issue 7, July-2016

[2] Parimala, P., and Raol, J. R. Image centroid tracking using square root information filter. International journal of electrical, electronics and data communication (IJEEDC), Vol. 4, No. $1, \quad$ pp. 31-34, Jan. 2016.www.ijeedc.iraj.in/ journal/jounrnal_file/journal pdf/1-221145499986031-34.pdf. (Approved by National Science Library ).

[3] Xiangdong Gao, Dukun Ding, Tianxiang Bai, and Seiji Katayama. Weld pool image centroid algorithm for seam tracking in arc welding process. IEEE paper, International Workshop on imaging systems and techniques, Shenzhen, China, May 11-12, 2009.

[4] Gerald J Bierman. An application of SRIF to large scale linear interconnected systems. Conference on Decision and Control, and Symposium on Adaptive Processes, and Special Symposium on Fuzzy Set Theory and Applications, New Orleans, La., Vol. 1. (A79-14957 04-63), Piscataway, N.J.,
Institute of Electrical and Electronics Engineers, Inc., pp. 930-933, December 7-9, 1977.

[5] S. Bruder. R, Johnson and M. Farooq. A multi-sensor tracking and identification algorithm using the square root information filter. IEEE Proceedings of Conference on Decision and Control, San Antonio, Texas, Vol. 1, pp. 940-941, December 15-17, 1993.

[6] V. P. S. Naidu, Girija G, and J. R. Raol. Data fusion for identity estimation and tracking of centroid using imaging sensor data. Defence Science Journal, Vol. 57, no. 5, pp. 639-652, September 2007. 